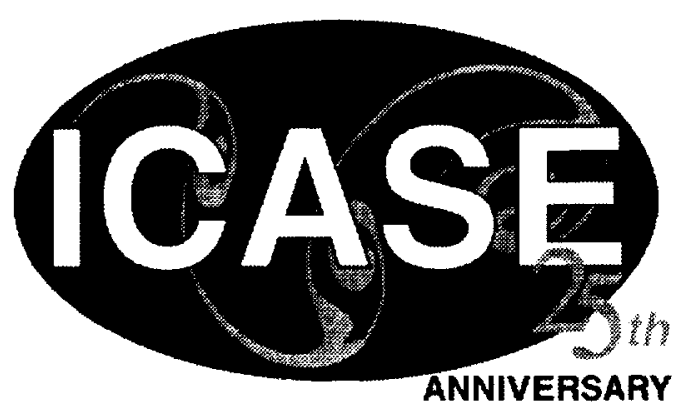

\title{
LQR CONTROL OF SHELL VIBRATIONS VIA PIEZOCERAMIC ACTUATORS
}

\section{R.C.H. del Rosario R.C. Smith}

NASA Contract No. NAS1-19480

March 1997

Institute for Computer Applications in Science and Engineering NASA Langley Research Center

Hampton, VA 23681-0001

Operated by Universities Space Research Association

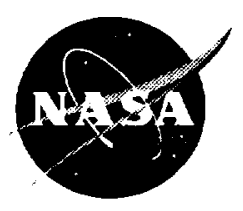

National Aeronautics and Space Administration

Langley Research Center Hampton, Virginia 23681-0001 



\title{
LQR CONTROL OF SHELL VIBRATIONS VIA PIEZOCERAMIC ACTUATORS ${ }^{1}$
}

\author{
R.(:.H. del Rosario \\ Center for Research in Scientific Computation \\ North ('arolina State University \\ Raleigh, No: 276955 \\ rcdelros eos.ncsu.edu \\ R.C. Smith \\ Department of Mathematics \\ Iowa State University \\ Ames, IA 50011 \\ rsmithoiastate.edu
}

\begin{abstract}
A model-based LQR method for controlling vibrations in cylindrical shells is presented. Surface-mounted piezoceramic patches are employed as actuators which leads to unbounded control input operators. Modified Donnell-Mushtari shell equations incorporating strong or Lelvin-Voigt damping are used to morlel the system. The model is then abstractly formulated in terms of sesquilinear forms. This provides a framework amenable for proving model wellposedness and convergence of LQR gains using analytic semigroup results combined with LQR theory for unbounded input operators. Finally, numerical examples clemonstrating the effectiveness of the method are presented.
\end{abstract}

\footnotetext{
'This research was supported in part by the National Aeronautios and Space Administration under NASA Contract Number NAS1-19480 while Re S' was a visiting scientist at the lnstit ute for (omputer Applications in Science and Engineering (ICASE), NASA Langley Research (enter, Hampton, VA 2:3681. Additional support was also provided in part under NASA grant NA(-1-1600.
} 



\section{Introduction}

The use of shell models to describe structural dynamics is pervasive in applications ranging from noise reduction in aircraft to flow control in flexible pipes. While general shell equations can be used in a variety of geometries, they all share the property that component displacements are coupled due to the geometry. This leads to significant challenges when developing appropriate models and approximation techniques, and constructing effective controllers.

In this paper, we consider cylindrical shells due to their prevalence in applications. Control is provided by piezoceramic patches bonded in pairs to the surface of the shell. These transducers provide significant actuating capabilities due to the piezoclectric effect in which input voltages generate strains in the patches. Utilization of the converse piezoelectric effect (strains produce voltages) also allows the patches to be employed as sensors. When combined with their light weight, space efficiency and reasonable cost, these properties make the patches highly effective control elements in a variety of applications. From a mathematical perspective, the use of surface-mounted piezoceramic patches leads to unbounded control input operators.

Experimental work has already demonstrated the potential for success when employing the patches as actuators in applications involving cylindrical shells $[8,13]$. However, these initial investigations have not, in general, utilized the full potential of the patches due to limitations in hardware. models, approximation techniques and control laws. For example. a common means of calculating control gains is through the use of modal expansions [10]. However, closed form expressions for the modes can be determined only for a limited set of models with severely restrictive boundary conditions. The use of incorrect modes when calculating control gains can lead to loss of control authority and possible controller instabilities. Hence for most physical shell models, modes must first be accurately approximated if modal methods are employed for control design.

In this paper, we present a model-based method for controlling shell vibrations. For simplicity, the Donnell-Mushtari shells equations with helvin-Voigt damping are used as a model (the assumption of strong or Kelvin-Voigt damping is reasonable and typical for many shell materials such as aluminum). The methods are general, however, and can be applied to higher-order models (e.g., Byrne-Flügge-Lur'ye model) if the application warrants. A general Galerkin method based on splines is then used to discretize the infinite dimensional system (see [7] for details regarding the numerical method and a comparison with finite element methods for shells). Through the choice of basis, the method is constructed to be flexible with regard to the boundary conditions and material nonhomogeneities which arise in typical applications. Furthermore, development of the model and approximation method in terms of a weak or energy formulation facilitates consideration of the distributional derivatives which arise when including patch contributions in the model. This provides a setting suitable for direct simulations and control design as well as computation of frequencies and modes for the shell.

The model and approximate system are then employed in an LQR full state feedback theory to obtain feedback gains and, ultimately, controlling voltages to the patches. While full state measurements are not available using current instrumentation, and hence the techniques cannot directly be implemented in experiments, they provide an important first step in the design of effective compensators based on state estimates calculated using a limited number of

obscrvations ( $\sec [5]$ ). The consideration of the LQR performance also illustrates properties of the system and model-based control techniques and facilitates investigations regarding issues 
such as patch number and configuration. Finally, the consideration of the problem provides a step toward the development of model-based controllers for fully coupled structural acoustic and fluid/structure systems involving cylindrical shells.

The strong and weak forms of the Donnell-Mushtari shells equations are outlined in Section 2. In presenting this model, care is taken to include both passive (material) and active (actuator) contributions due to the patches. An abstract form of the model, based on sesquilinear forms, is also presented. This provides a natural setting to prove model well-posedness and convergence properties of the LQR control law. LQR full state feedback laws for systems with no exogenous force or forces which are periodic in time are presented in Section 3. In the former case, convergence of the approximate suboptimal gains to the optimal gains for the infinite dimensional system is proven using analytic semigroup theory in combination with LQR results for unbounded control input operators. A Fourier-Galerkin method for approximating the system dynamics is outlined in Section 4, and the effectiveness of the LQR method for periodic forces is demonstrated through a numerical example in Section 5. This example demonstrates that through the use of the model-based methodology with general (ialerkin approximations, significant attenuation in shell vibrations can be obtained using piezoceramic patches.

\section{PDE Model}

The system under consideration consists of a thin cylindrical shell with surface-mounted piezoceramic patches. It is assumed that the patches are mounted in pairs with edges aligned with the circumferential and longitudinal axes of the shell. The edges of the shell are taken to be fixed in accordance with common experimental clamping techniques.

To specify the geometry for the corresponding model, we consider the longitudinal direction to be aligned along the $x$-axis as depicted in Figure 1. The displacements of the middle surface in the longitudinal, circumferential and transverse directions are denoted by $u, v$ and $w$. respectively while the length. thickness and radius of the shell are denoted by $t, h, R$. The region occupied by the middle surface is denoted by $\Gamma_{0}$. Finally, the shell is assumed to have mass density $\rho$, Young 's modulus $E$, Poisson ratio $\nu$, Kelvin-Voigt damping coefficient $c_{D}$ and air damping coefficient $\mu$.

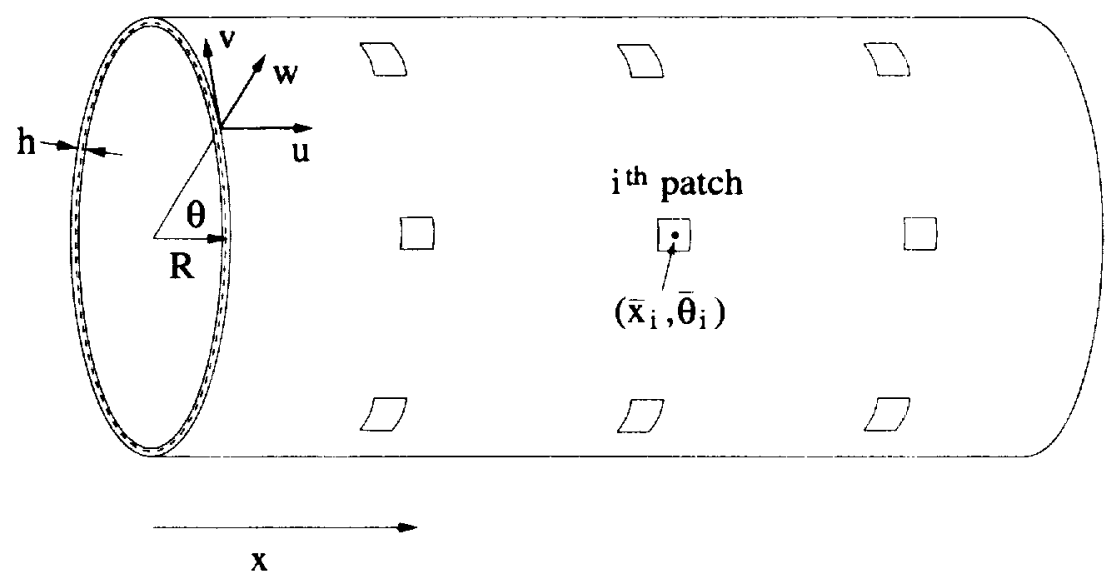

Figure 1. Thin cylindrical shell with surface monnted piezoceramic patches. 
Actuator and/or sensor capabilities are provided by $s$ pairs of surface-mounted piezoceramic patches. It is assumed that all the patches have thickness $h_{p \epsilon}$, Young's modulus $E_{p e}$, Poisson ratio $\nu_{p \epsilon}$ and Kelvin-Voigt damping coefficient $c_{D_{p e}}$. Furthermore, it is assumed that the glue bonding layer provides negligible contribution to the structural dynamics (the reader is referred to $[3,5]$ for details concerning the incorporation of differing patch characteristics and bonding layers in the ensuing models). The region covered by the $i^{\text {th }}$ patch pair, with edges at $x_{1 i}, x_{2 i}, \theta_{1 i}, \theta_{2 i}$, is delineated by the characteristic function

$$
\gamma_{p \epsilon_{i}}(x, \theta)= \begin{cases}1, & x_{1 i} \leq x \leq x_{2 i}, \theta_{1 i} \leq \theta \leq \theta_{2 i} \\ 0, & \text { otherwise }\end{cases}
$$

The indicator function $\mathcal{S}_{p r_{2}}(x, \theta) \equiv S_{1,2}(x) \hat{S}_{1.2}(\theta)$, where

$$
S_{1,2}(x)=\left\{\begin{array}{cll}
1 & , & x<\left(x_{1 i}+x_{2 i}\right) / 2 \\
0 & , & x=\left(x_{1 i}+x_{2 i}\right) / 2 \\
-1 & , & x>\left(x_{1 i}+x_{2 i}\right) / 2
\end{array} \quad, \quad \hat{S}_{1,2}(\theta)=\left\{\begin{array}{cc}
1, & \theta<\left(\theta_{1 i}+\theta_{2 i}\right) / 2 \\
0, & \theta=\left(\theta_{1 i}+\theta_{2 i}\right) / 2 \\
-1, & \theta>\left(\theta_{1 i}+\theta_{2 i}\right) / 2
\end{array},\right.\right.
$$

delineates the sense of the forces generated by the $i^{\text {th }}$ pair. The symmetry of the function arises from the property that for homogeneous patches having uniform thickness, equal but opposite strains are generated about the point $\left(x_{i}, \dot{\theta}_{i}\right)=\left(\left(x_{1 i}+x_{2 i}\right) / 2,\left(\theta_{1 i}+\theta_{2 i}\right) / 2\right)$.

\subsection{Strong Form of the Modeling Equation}

We consider here the modified Donnell-Mushtari equations

$$
\begin{gathered}
R \rho h \frac{\partial^{2} u}{\partial t^{2}}-R \frac{\partial N_{x}}{\partial x}-\frac{\partial N_{\theta x}}{\partial \theta}=R \hat{q}_{x}-R \sum_{i=1}^{s} \frac{\partial\left(N_{x}\right)_{p \epsilon_{i}}}{\partial x} \mathcal{S}_{p \epsilon_{i}}(x, \theta) \\
R \rho h \frac{\partial^{2} r}{\partial t^{2}}-\frac{\partial N_{\theta}}{\partial \theta}-R \frac{N_{x \theta}}{\partial x}=R \hat{q}_{\theta}-\sum_{i=1}^{s} \frac{\partial\left(N_{\theta}\right)_{p t_{i}}}{\partial \theta} \mathcal{S}_{p \epsilon_{i}}(x, \theta) \\
R \rho h \frac{\partial^{2} u}{\partial t^{2}}+R \mu \frac{\partial w}{\partial t}-R \frac{\partial^{2} M_{x}}{\partial x^{2}}-\frac{1}{R} \frac{\partial^{2} M_{\theta}}{\partial \theta^{2}}-2 \frac{\partial^{2} M_{x \theta}}{\partial x \partial \theta}+N_{\theta} \\
=R \hat{q}_{n}-\sum_{i=1}^{s}\left[R \frac{\partial^{2}\left(M_{x}\right)_{p \epsilon_{i}}}{\partial x^{2}}+\frac{1}{R} \frac{\partial^{2}\left(M_{\theta}\right)_{p \epsilon_{i}}}{\partial \theta^{2}}\right]
\end{gathered}
$$

as a model for the thin shell dynamics. As detailed in $[3,5,9]$, these equations are obtained through force and moment balancing with only low order terms retained. Here $M_{x}, M_{\theta}, M_{\theta x}$ and $M_{x \theta}$ are internal moments while $N_{2}, N_{\theta}, N_{\theta x}$ and $N_{x \theta}$ denote internal force resultants. External surface forces are denoted by $\hat{q}_{x}, \hat{q}_{\theta}, \hat{q}_{n}$ whereas the external resultants (line moments and forces) generated by the $i^{\text {th }}$ patch pair are designated by $\left(M_{r}\right)_{p r_{1},}\left(M_{\theta}\right)_{p r_{i}},\left(N_{x}\right)_{p r_{i},}\left(N_{\theta}\right)_{p \epsilon_{i}}$.

Expressions for the internal force and moment resultants are derived under the assumption that stress is proportional to a linear combination of strain and strain rate. This yields a model which incorporates Kielvin-Voigt or strong internal damping. As detailed in [3, 5], the 
resultants $N_{x}, N_{x \theta}, N_{\theta x}, M_{x}, M_{x \theta}, M_{\theta x}$ derived under this assumption are

$$
\begin{aligned}
& N_{s}=\frac{E h}{1-\nu^{2}}\left(\varepsilon_{x}+\nu \varepsilon_{\theta}\right)+\sum_{i=1}^{s} \frac{2 E_{p \epsilon} h_{p \epsilon}}{1-\nu_{p e}^{2}}\left(\varepsilon_{x}+\nu_{p e} \varepsilon_{\theta}\right) \gamma_{p \epsilon_{i}}(x, \theta)
\end{aligned}
$$

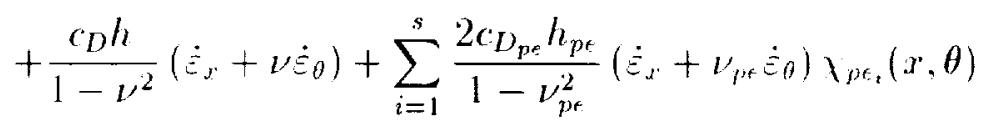

$$
\begin{aligned}
& N_{x \theta}=N_{\theta r}=\frac{k h}{2(1+\nu)} \varepsilon_{x \theta}+\sum_{i=1}^{s} \frac{E_{p e} h_{p r}}{\left(1+\nu_{p e}\right)} \varepsilon_{x \theta} \backslash p_{1}(r, \theta) \\
& +\frac{c_{p} h}{2(1+\nu)} \dot{\varepsilon}_{x \theta}+\sum_{i=1}^{s} \frac{c_{D_{p^{m}}} h_{p_{r}}}{\left(1+\nu_{p e}\right)} \dot{\varepsilon}_{x \theta} \backslash p^{\prime \prime}(x, \theta) \\
& M_{x}=\frac{E h^{3}}{12\left(1-\nu^{2}\right)}\left(r_{,}+\nu \kappa_{\theta}\right)+\sum_{i=1}^{s} \frac{2 E_{p r} a_{3}}{3\left(1-\nu_{p s}^{2}\right)}\left(r_{\nu}+\nu_{p r} \kappa_{\theta}\right) \lambda_{p r_{2}}(x, \theta) \\
& +\frac{r_{\nu} h^{3}}{12\left(1-\nu^{2}\right)}\left(\dot{r}_{x}+\nu \dot{r}_{\theta}\right)+\sum_{i=1}^{s} \frac{2 c_{D_{\mu}} a_{3}}{3\left(1-\nu_{p t}^{2}\right)}\left(\dot{r}_{x}+\nu_{p+} \dot{r}_{\theta}\right) \nu_{\nu}(x, \theta) \\
& M_{x \theta}=M_{\theta x}=\frac{E h^{3}}{21(1+\mu)} \tau+\sum_{i=1}^{s} \frac{E_{p r} a_{3}}{3\left(1+\nu_{p^{*}}\right)} \tau \chi_{\mu^{\prime},}(x, \theta) \\
& +\frac{{ }_{p} h^{3}}{24(1+\nu)} \dot{\tau}+\sum_{i=1}^{s} \frac{c_{D_{r,}} a_{3}}{3\left(1+\nu_{p \epsilon}\right)} \dot{\tau} \gamma_{\mu_{i}}(r . \theta)
\end{aligned}
$$

where the constant $a_{3} \equiv\left(h / 2+h_{p}\right)^{3}-h^{3} / 8$ results from integration through the thickness of the patch. Expressions for the resultants $N_{\theta}$ and $M_{\theta}$ can be obtained by replacing $\varepsilon_{j}$, $\varepsilon_{\theta}, k_{x}$, tio in the expressions for $N_{x}$ and $M_{x}$ by $\varepsilon_{\theta}, \varepsilon_{x}, \kappa_{\theta}, \kappa_{x}$. respectively. The midsurface strains and changes in curvature for the Donmell-Mushtari model are

$$
\begin{array}{lll}
\varepsilon_{x}=\frac{\partial u}{\partial x}, & \varepsilon_{\theta}=\frac{1}{R} \frac{\partial v}{\partial \theta}+\frac{w}{R} . & \varepsilon_{\theta}=\frac{\partial v}{\partial x}+\frac{1}{R} \frac{\partial u}{\partial \theta} \\
\kappa_{x}=-\frac{\partial^{2} u}{\partial x^{2}} \quad . \quad \kappa_{\theta}=-\frac{1}{R^{2}} \frac{\partial^{2} w}{\partial \theta^{2}} \quad . \quad \tau=-\frac{2}{R} \frac{\partial^{2} w}{\partial x \partial \theta} .
\end{array}
$$

Note that for the undamped shell which is devoid of patches, the resultant equations (2.2) reduce to the classical Donnell-Mushtari expressions

$$
\begin{aligned}
& N_{x}=\frac{E h}{\left(1-v^{2}\right)}\left[\frac{\partial u}{\partial x}+\frac{\nu}{R}\left(\frac{\partial v}{\partial \theta}+w\right)\right], \quad M_{x}=\frac{-E h^{3}}{12\left(1-v^{2}\right)}\left[\frac{\partial^{2} w}{\partial x^{2}}+\frac{\nu}{R^{2}} \frac{\partial^{2} w}{\partial \theta^{2}}\right] \\
& N_{\theta}=\frac{E h}{\left(1-\nu^{2}\right)}\left[\frac{1}{R} \frac{\partial u}{\partial \theta}+\frac{u^{\prime}}{R}+\nu \frac{\partial u}{\partial x}\right] . \quad M_{\theta}=\frac{-E h^{3}}{12\left(1-\nu^{2}\right)}\left[\frac{1}{R^{2}} \frac{\partial^{2} w}{\partial \theta^{2}}+\nu \frac{\partial^{2} w}{\partial x^{2}}\right] \\
& N_{x \theta}=N_{\theta x}=\frac{E h}{2(1+\nu)}\left[\frac{\partial u^{\prime}}{\partial x}+\frac{1}{l} \frac{\partial u}{\partial \theta}\right], \quad M_{x \theta}=M_{\theta x}=\frac{-E h^{3}}{12 R(1+\nu)} \frac{\partial^{2} w}{\partial x \partial \theta}
\end{aligned}
$$

(e.g., see $[9]$ ). 
To characterize the external or active patch contributions, it is typical to start with the assumption that the strains generated by a patch are proportional to the applied voltage [3]. Since differing voltages can be applied to the outer and inner patches in the pair, we will differentiate between the two with $V_{i 1}(t)$ and $V_{i 2}(t)$ used to denote the voltages to the outer and inner patches in the $i^{\text {th }}$ pair, respectively. The proportionality constant relating the generated strain to the input voltage is designated by $d_{31}$. As detailed in [3], the total external moments and forces generated by the patches are

$$
\begin{aligned}
& \left(M_{s}\right)_{p \epsilon_{i}}=\frac{-E_{p \epsilon}}{1-\nu_{p \epsilon}} \cdot \frac{d_{31}}{h_{p \epsilon}} \lambda_{p \epsilon_{2}}(x, \theta)\left[\left(\frac{a_{2}}{2}+\frac{a_{3}}{3 R}\right) V_{i 1}-\left(\frac{a_{2}}{2}-\frac{a_{3}}{3 R}\right) V_{i 2}\right] \\
& \left(M_{\theta}\right)_{p \epsilon_{\imath}}=\frac{-E_{p \epsilon}}{1-\nu_{p \epsilon}} \cdot \frac{d_{31} a_{2}}{2 h_{p \epsilon}} \backslash_{p \epsilon_{i}}(x, \theta)\left[V_{i 1}-V_{i 2}\right] \\
& \left(N_{x}\right)_{p r_{i}}=\frac{-E_{p \epsilon}}{1-\nu_{p \epsilon}} \cdot \frac{d_{31}}{h_{p \epsilon}} \backslash_{p *_{i}}(x, \theta) \mathcal{S}_{p c_{1}}(x, \theta)\left[\left(h_{p \epsilon}+\frac{a_{2}}{2 R}\right) V_{i 1}+\left(h_{p \epsilon}-\frac{a_{2}}{2 R}\right) V_{i 2}\right] \\
& \left(N_{\theta}\right)_{p \epsilon_{i}}=\frac{-E_{p \epsilon}}{1-\nu_{p r}} d_{31 \backslash p \epsilon_{2}}(x, \theta) \mathcal{S}_{p \epsilon_{i}}(x, \theta)\left[V_{i 1}-V_{i 2}\right]
\end{aligned}
$$

where $a_{2}=\left(h / 2+h_{p \epsilon}\right)^{2}-h^{2} / 4$ and $a_{3}=\left(h / 2+h_{p e}\right)^{3}-h^{3} / 8$. When substituted into (2.1), the expressions $(2.4)$ provide the input from the patches when voltages are applied.

Finally, the fixed-edge boundary conditions

$$
u=v=w=\frac{d w}{\partial x}=0 \quad, \quad x=0, \ell
$$

are used to model the end behavior of the shell. These boundary conditions are appropriate for experimental setups in which heavy endcaps prevent edge movement. Note that alternative boundary conditions such as simply supported or "almost fixed" (see [4]) can be employed if edge movement is suspected.

\subsection{Weak Form of Modeling Equations}

The strong form (2.1) of the modeling equations requires first and second derivatives of the moment and force resultants. As noted in (2.2) and (2.4), both the internal and external moment and force resultants are discontinuous due to the piezoceramic patches. Hence formal analysis and approximation using the strong form of the modeling equations lead to difficulties due to differentiation of Dirac distributions.

To alleviate these difficulties, it is advantageous to consider a weak form of the modeling equations which can be derived from Hamilton's principle (energy considerations). While equivalent to the strong form under suitable smoothness assumptions, the weak form provides a more natural setting for analysis and approximation.

The state variables for the problem in second-order form are taken to be $y=(u, v, w)$ in the state space $H=L^{2}\left(\Gamma_{0}\right) \times L^{2}\left(\Gamma_{0}\right) \times L^{2}\left(\Gamma_{0}\right)$. For the fixed-edge boundary conditions (2.5), the space of test functions is taken to be $V=H_{0}^{1}\left(\Gamma_{0}\right) \times H_{0}^{1}\left(\Gamma_{0}\right) \times H_{0}^{2}\left(\Gamma_{0}\right)$ where

$$
\begin{aligned}
& H_{0}^{1}\left(\Gamma_{0}\right)=\left\{\eta \in H^{1}\left(\Gamma_{0}\right) \mid \eta(0, \theta)=\eta(f, \theta)=0\right\} \\
& H_{0}^{2}\left(\Gamma_{0}\right)=\left\{\eta \in H^{2}\left(\Gamma_{0}\right) \mid \eta(0, \theta)=\eta_{x}(0, \theta)=\eta(\ell, \theta)=\eta_{x}(\ell, \theta)=0\right\} .
\end{aligned}
$$


For $\Phi=(u, v, w)$ and $\Psi=\left(\eta_{1}, \eta_{2}, \eta_{3}\right)$, the $H$ and $V$ inner products are taken to be

$$
\langle\Phi, \Psi\rangle_{H}=\int_{\Gamma_{0}} \rho h u \bar{\eta}_{1} d \gamma+\int_{\Gamma_{0}} \rho h v \bar{\pi}_{2} d \gamma+\int_{\Gamma_{0}} \rho h \omega \bar{\eta}_{3} d \gamma
$$

and

$$
\begin{aligned}
& \left\langle\left(E, E_{p \epsilon}\right) \Phi, \Psi\right\rangle_{V}=\int_{\Gamma_{0}}\left\{\frac{E h}{1-\nu^{2}}\left[\left(\varepsilon_{x}+\nu \varepsilon_{\theta}\right) \frac{\overline{\partial \eta_{1}}}{\partial x}+\frac{1}{2 R}(1-\nu) \varepsilon_{\alpha} \theta \frac{\overline{\partial \eta_{1}}}{\partial \theta}\right]\right. \\
& \left.+\sum_{i=1}^{s} \frac{2 E_{p r} h_{p r}}{1-\nu_{p r}^{2}} \backslash_{p_{c}}(x, \theta)\left[\left(\varepsilon_{x}+\nu_{p r} \varepsilon_{\theta}\right) \frac{\overline{\partial \eta_{1}}}{\partial x}+\frac{1}{2 h}\left(1-\nu_{p c}\right) \varepsilon_{r \theta} \frac{\overline{\partial \eta_{1}}}{\partial \theta}\right]\right\} d \gamma \\
& +\int_{\Gamma_{0}}\left\{\frac{E h}{1-\nu^{2}}\left[\left(\varepsilon_{\theta}+\nu \varepsilon_{x}\right) \frac{\overline{\partial \eta_{1}}}{\partial \theta}+\frac{1}{2 R}(1-\nu) \varepsilon_{x \theta} \frac{\overline{\partial \eta_{2}}}{\partial x}\right]\right. \\
& \left.+\sum_{i=1}^{s} \frac{2 E_{p r} h_{p r}}{1-\nu_{p r}^{2}} \gamma_{p c_{i}}(x, \theta)\left[\left(\varepsilon_{\theta}+\nu_{p r} \varepsilon_{x}\right) \frac{\overline{\partial \eta_{2}}}{\partial \theta}+\frac{1}{2 R}\left(1-\nu_{p r}\right) \varepsilon_{x \theta} \frac{\overline{\partial \eta_{2}}}{\partial x}\right]\right\} d \gamma \\
& +\int_{\Gamma_{0}}\left\{\frac { L h } { 1 - l ^ { \prime 2 } } \left[\frac{1}{h}\left(\varepsilon_{\theta}+\nu \varepsilon_{x}\right) \overline{\eta_{3}}-\frac{h^{2}}{12}\left(\kappa_{x}+\nu \kappa_{\theta}\right) \frac{\overline{\partial^{2} \eta_{3}}}{\partial x^{2}}\right.\right. \\
& \left.-\frac{h^{2}}{12 h^{2}}\left(h_{\theta}+\nu \kappa_{x}\right) \frac{\overline{\partial^{2} \eta_{3}}}{\partial \theta^{2}}-\frac{h^{2}}{12 R}(1-\nu) \tau \frac{\overline{\partial^{2} \eta_{3}}}{\partial \partial_{2} \partial \theta}\right] \\
& +\sum_{i=1}^{s} \frac{2 L_{p r}}{3\left(1-\nu_{p e}^{2}\right)} \gamma_{p e}(x, \theta)\left[\frac{3 h_{p e}}{R}\left(\varepsilon_{\theta}+\nu_{p t} \Sigma_{j}\right) \overline{\eta_{i 3}}-a_{3}\left(\kappa_{x}+\nu_{p e} \kappa_{\theta}\right) \frac{\overline{\partial^{2} \eta_{3}}}{\partial x^{2}}\right. \\
& \left.\left.-\frac{a_{3}}{R^{2}}\left(r_{\theta}+\nu_{p \epsilon} \kappa_{\alpha}\right) \overline{\frac{\partial^{2} \eta_{3}}{\partial \theta^{2}}}-\frac{a_{3}}{R}\left(1-\nu_{p \epsilon}\right) \tau \overline{\frac{\partial^{2} \eta_{3}}{\partial \partial_{\alpha} \partial \theta}}\right]\right\} d \gamma
\end{aligned}
$$

where $\Sigma_{x}, \Sigma_{\theta}, \varepsilon_{x \theta}, \kappa_{x}, \kappa_{\theta}, \tau$ are defined in $(2.3)$ and $d_{\gamma}=R d \theta d . r$. The dependence of the inmer product on the Young's moduli is explicitly included in the definition to provide a notation for defining analogous damping expressions later in this work.

The weak form of (2.1), as derived in [5] from energy principles, is given by

$$
\begin{aligned}
& \int_{\Gamma_{0}}\left\{R \rho h \frac{\partial^{2} u}{\partial t^{2}} \bar{\eta}_{1}+R N, \frac{\overline{\partial \eta_{1}}}{\partial x}+N_{\theta x} \frac{\overline{\partial \eta_{1}}}{\partial \theta}-R \hat{\eta}_{r} \bar{\eta}_{1}-R \sum_{i=1}^{s}\left(N_{x}\right)_{p c_{i}} \frac{\overline{\partial \eta_{1}}}{\partial x}\right\} d \gamma=0 \\
& \int_{\Gamma_{0}}\left\{R \rho h \frac{\partial^{2} v}{\partial t^{2}} \bar{\eta}_{2}+N_{\theta} \frac{\overline{\partial \eta_{2}}}{\partial \theta}+R N_{r \theta} \frac{\overline{\partial \eta_{2}}}{\partial x}-R \hat{q}_{\theta} \bar{\eta}_{2}-\sum_{i=1}^{s}\left(N_{\theta}\right)_{p} \frac{\overline{\partial \eta_{2}}}{\partial \theta}\right\} d \gamma_{\gamma}=0 \\
& \int_{\Gamma_{0}}\left\{R \rho h \frac{\partial^{2} u}{\partial l^{2}} \bar{\eta}_{3}+R \mu \frac{\partial u}{\partial t} \bar{\eta}_{3}+N_{\theta} \bar{\eta}_{3}-R M_{2} \frac{\overline{\partial^{2} \eta_{3}}}{\partial x^{2}}-\frac{1}{R} M_{\theta} \frac{\overline{\partial^{2} \eta_{3}}}{\partial \theta^{2}}-2 M_{x \theta} \frac{\overline{\partial^{2} \eta_{3}}}{\partial x \partial \theta}\right. \\
& \left.-R \hat{q}_{n} \bar{\eta}_{3}+\sum_{i=1}^{s}\left[R\left(M_{s}\right)_{p+,} \frac{\overline{\partial^{2} \eta_{3}}}{\partial x^{2}}+\frac{1}{R}\left(M_{\theta}\right)_{p,} \frac{\overline{\partial^{2} \eta_{3}}}{\partial \theta^{2}}\right]\right\} d h=0
\end{aligned}
$$


for all $\Psi=\left(\eta_{1}, \eta_{2}, \eta_{3}\right) \in V$. A comparison between (2.6) and (2.1) illustrates that in the weak form, derivatives are transferred from the discontinuous resultants onto suitably smooth test functions. This alleviates the difficulties associated with the discontinuities and reduces smoothness requirements on approximate solutions.

\subsection{Abstract Formulation}

To define appropriate sesquilinear forms, we group stiffness components separately from damping components. To this end, we define $\sigma_{i}: V \times V \rightarrow \mathbb{C}, i=1,2$ by

$$
\begin{aligned}
& \sigma_{1}(\Phi, \Psi)=\left\langle\left(E, E_{p e}\right) \Phi, \Psi\right\rangle_{V} \\
& \sigma_{2}(\Phi, \Psi)=\left\langle\left(c_{D}, c_{D_{p}}\right) \Phi, \Psi\right\rangle_{V}+\int_{\Gamma_{0}} \mu u \bar{\eta}_{3} d \gamma
\end{aligned}
$$

Note that $\left\langle\left(c_{D}, C_{p^{\prime}}\right) \Phi, \Psi\right\rangle_{V}$ differs from $\left\langle\left(E, E_{p \epsilon}\right) \Phi, \Psi\right\rangle_{V}$ only in that Young's moduli are replaced by Kelvin-Voigt damping coefficients. It can be directly verified that the stiffness form $\sigma_{1}$ satisfies

$$
\begin{array}{ll}
\left|\sigma_{1}(\Phi, \Psi)\right| \leq c_{1}|\Phi|_{V}|\Psi|_{V}, \text { for some } c_{1} \in \mathbb{R} & \text { (Bounded) } \\
\operatorname{Re} \sigma_{1}(\Phi, \Phi) \geq c_{2}|\Phi|_{V}^{2}, \text { for some } c_{2}>0 & \text { (V-Elliptic) } \\
\sigma_{1}(\Phi, \Psi)=\overline{\sigma_{1}(\Psi, \Phi)} & \text { (Symmetric) }
\end{array}
$$

for all $\Phi, \Psi \in V$. Moreover, the damping term $\sigma_{2}$ satisfies

$$
\begin{array}{ll}
\left|\sigma_{2}(\Phi, \Psi)\right| \leq c_{3}|\Phi|_{V}|\Psi|_{V}, \text { for some } c_{3} \in \mathbb{R} & \text { (Bounded) } \\
\operatorname{Re} \cdot \sigma_{2}(\Phi, \Phi) \geq c_{4}|\Phi|_{V}^{2}, \text { for some } c_{4}>0 & \text { (V-Elliptic) }
\end{array}
$$

Remark 1. The symmetry of $\sigma_{1}$ is dependent upon the choice of shell model and ultimately reflects the Maxwell-Betti reciprocity theorem. While the Donnell-Mushtari model yields a symmetric sesquilinear form $\sigma_{1}$, other models such as the Timoshenko shell model will not yield a symmetric form.

To represent control contributions, let $U=\mathbb{R}^{s}$ denote the Hilbert space of control inputs and define $B \in \mathcal{L}\left(U, V^{*}\right)$ by

$$
\begin{aligned}
& \langle B u(t), \Psi\rangle_{V^{*}, l^{\prime}}=\int_{\Gamma_{0}} \sum_{i=1}^{s}\left\{\left(N_{x}\right)_{p \epsilon_{i}} \frac{\overline{\partial \eta_{1}}}{\partial x}+\frac{1}{R}\left(N_{\theta}\right)_{p r_{i}} \frac{\overline{\partial \eta_{2}}}{\partial \theta}\right. \\
& \left.-\left(M_{x}\right)_{p \epsilon_{i}} \frac{\overline{\partial^{2} \eta_{3}}}{\partial x^{2}}-\frac{1}{R^{2}}\left(M_{\theta}\right)_{p \epsilon_{i}} \overline{\frac{\partial^{2} \eta_{3}}{\partial \theta^{2}}}\right\} d \gamma
\end{aligned}
$$

for $\Psi \in V$. Here $\langle\cdot \cdot\rangle_{V *}$. denotes the usual duality product. Finally, with the definition $\tilde{g}=(1 / \rho h)\left[\hat{q}_{2}, \hat{q}_{\theta}, \hat{q}_{n}\right]$, we can write the weak form (2.6) in the abstract variational form

$$
\langle\ddot{y}(t), \Psi\rangle_{1 \cdot .}+\sigma_{2}(\dot{y}(t), \Psi)+\sigma_{1}(y(t), \Psi)=\langle B u(t)+\dot{g}(t), \Psi\rangle_{1 \cdot . l} .
$$


To pose the problem in a first-order form amenable for control applications, we define the product spaces $\mathcal{H}=V \times H$ and $\mathcal{V}=V \times V$ with the norms

$$
\begin{aligned}
& \left|\left(\phi_{1}, \phi_{2}\right)\right|_{\mathcal{H}}^{2}=\left|\phi_{1}\right|_{V}^{2}+\left|\phi_{2}\right|_{H}^{2} \\
& \left|\left(\phi_{1}, \phi_{2}\right)\right|_{V}^{2}=\left|\phi_{1}\right|_{V}^{2}+\left|\phi_{2}\right|_{V}^{2} .
\end{aligned}
$$

The state is taken to be $z(l)=(y(t), \dot{y}(t)) \in \mathcal{H}$. Finally, the product space forcing terms are formulated as

$$
g(t)=\left[\begin{array}{c}
0 \\
g(t)
\end{array}\right] \quad, \quad \mathcal{B} u(t)=\left[\begin{array}{c}
0 \\
B u(t)
\end{array}\right] .
$$

The weak form (2.8) can then be rewritten as

$$
\begin{aligned}
& \langle\dot{z}(t), \Lambda\rangle_{\mathcal{V}^{*}, \mathcal{V}}+\sigma(z(t), \Lambda)=\langle\mathcal{B} u(l)+g(t), \Lambda\rangle_{\mathcal{V} * \mathcal{V}} \quad \text { for } \Lambda \in \mathcal{V} \\
& z(0)=z_{0}=\left(y_{0}, y_{1}\right)
\end{aligned}
$$

where $\sigma: \mathcal{V} \times \mathcal{V} \rightarrow \mathbb{Q}:$ is given by

$$
\sigma\left(\phi, \iota^{\prime}\right)=-\left\langle\phi_{2}, \psi_{1}\right\rangle_{1}+\sigma_{1}\left(\phi_{1}, \psi_{2}\right)+\sigma_{2}\left(\phi_{2}, \iota_{2}\right)
$$

for $\phi=\left(\phi_{1}, \phi_{2}\right), \psi^{\prime}=\left(\psi_{1}, \psi_{2}\right) \in \mathcal{V}$. As proven in [5, page 109], $\sigma$ is $\mathcal{V}$ continuous and for $\lambda>0, \sigma(\cdot, \cdot)+\lambda\langle\cdot, \cdot\rangle_{H}$ is $\mathcal{V}$-elliptic. From the continuity of $\sigma$. it follows that one can define all operator $\tilde{\mathcal{A}} \in \mathcal{L}\left(\mathcal{V}, \mathcal{V}^{*}\right)$ by $\sigma(\Upsilon, \Lambda)=\langle\tilde{\mathcal{A}} \Upsilon, \Lambda\rangle_{\mathcal{V}^{*}, \mathcal{V}}$

To obtain a strong form of the first-order system which is appropriate for control purposes, consider the system operator

$$
\begin{aligned}
& \operatorname{dom} \mathcal{A}=\left\{\left(\phi_{1}, \phi_{2}\right) \in \mathcal{H} \mid \phi_{2} \in V . A_{1} \phi_{1}+A_{2} \phi_{2} \in H\right\} \\
& \mathcal{A}=\left[\begin{array}{cc}
0 & I \\
-A_{1} & -A_{2}
\end{array}\right]
\end{aligned}
$$

with $A_{1}, A_{2} \in \mathcal{L}\left(V, V^{*}\right)$ defined by

$$
\left\langle A_{i} \phi_{1}, \phi_{2}\right\rangle_{1 *, 1}=\sigma_{i}\left(\phi_{1}, \phi_{2}\right) \quad, \quad i=1,2 .
$$

It should be notated that $\mathcal{A}$ is the negative of the restriction to $\operatorname{dom} \mathcal{A}$ of $\tilde{\mathcal{A}} \in \mathcal{L}\left(\mathcal{V}\right.$. $\left.\mathcal{V}^{*}\right)$ so that $\sigma(\Upsilon, \Lambda)=\langle-\mathcal{A} \Upsilon, \Lambda\rangle_{\mathcal{H}}$ for $\Upsilon \in \operatorname{dom} \mathcal{A}, \Lambda \in \mathcal{V}$.

A strong form of the alsitract system model is then given by

$$
\begin{aligned}
& \dot{z}(t)=\mathcal{A} z(t)+\mathcal{B} u(t)+g(t) \quad \text { in } \mathcal{V}^{*}=V \times V^{*} \\
& z(0)=z_{0} .
\end{aligned}
$$

The rigorous equivalence of solutions is established through the following theorems.

Theorem 1. Inder Hypotheses (III)-(H5) on $\sigma_{1}$ and $\sigma_{2}$. $\mathcal{A}$ generates an analytic semigroup) $\mathcal{T}(t)$ on $\mathcal{V}, \mathcal{H}$ and $\mathcal{V}^{*}$. In terms of this semigroup, the representation

$$
z(t)=\mathcal{T}(t) \approx 0+\int_{0}^{t} \mathcal{T}(t-s)[\mathcal{B} u(s)+g(s)] d s
$$

defines a mild solution to (2.12) for $z_{0} \in \mathcal{V}^{*}$ and $\mathcal{B} u+g \in L^{2}\left(\left(0, T^{\prime}\right) ; \mathcal{V}^{*}\right)$. Furthermore, this semigroup is (uniformly) exponent ially stable on $\mathcal{V} . \mathcal{H}$ and $\mathcal{V}^{*}$. 
Theorem 2. Let $z_{s g}$ denote the semigroup solution to (2.12) given by $(2.13)$ and let $v_{\text {var }}$ denote the weak solution to (2.8). Under hypotheses (II1)-(II5), it follows that $z_{s g}\left(z_{0}, \mathcal{F}\right)=z_{\text {var }}\left(z_{0}, \mathcal{F}\right)$ for $\tilde{z}_{0} \in \mathcal{H}$ and $\mathcal{F} \equiv \mathcal{B} u+g \in L^{2}\left((0, T) ; \mathcal{V}^{*}\right)$.

Following the convention of [14], we will use the same notation for the semigrou]s defined on $\mathcal{V}, \mathcal{H}$ and $\mathcal{V}^{*}$ since each semigroup is an extension or restriction of the others. Note that $\operatorname{dom} \mathcal{A}$ defined in $(2.11)$ is actually $\operatorname{dom}_{\mathcal{H}} \widetilde{\mathcal{A}}$, the domain of $\tilde{\mathcal{A}}$ as a generator of $\mathcal{T}(t)$ in $\mathcal{H}$. As detailed in Lemma 3.6.1 and Theorem 3.6.1 of [14] (sec also Section IV.6 of [12] and Chapter 2, Theorem 5.2 of [11]), the property that $\tilde{\mathcal{A}}$ generates an analytic semigroup on $\mathcal{V}, \mathcal{H}$ and $\mathcal{V}^{*}$ results from the continuity and $\mathcal{V}$-ellipticity of $\sigma$. The exponential stability of $\mathcal{T}(t)$ on $\mathcal{H}$ for second-order systems with strong damping is demonstrated in [1] while the exponential stability of $\mathcal{T}(t)$ on $\mathcal{V}$ and $\mathcal{V}^{*}$ in this setting is proven in Lemma 3.3 of [2]. Finally, Theorem 2 is a reformulation of Theorem 4.14 of $[5]$ and details can be found therein.

\section{LQR Control Problem}

In the last section, the PDE system modeling the dynamics of the thin shell with surfacemounted piezoceramic actuators was written in the abstract first-order form

$$
\begin{aligned}
& \dot{z}(t)=\mathcal{A} \approx(t)+\mathcal{B} u(t)+g(t) \\
& \approx(0)=z_{0}
\end{aligned}
$$

in $\mathcal{V}^{*}$. In this section, LQR control results for both the original infinite dimensional problem and approximating finite dimensional problems will be discussed. 'Two cases will be considered, namely when $g \equiv 0$ and $g$ is periodic in time. In both cases, it is assumed that state observations in an observation space $Y$ have the form

$$
\tilde{z}_{o b}(t)=\mathcal{C} \approx(t)
$$

where $\mathcal{C} \in \mathcal{L}(\mathcal{H}, Y)$ is bounded. The assumption that $\mathcal{C}$ is bounded is marle to simplify the exposition and the reader is referred to [2] for arguments pertaining to the case of unbounded observation operators.

\subsection{No Exogenous Input}

For the case in which $g \equiv 0$, the infinite horizon problem concerns the determination of a control $u$ which minimizes the quadratic cost functional

$$
J\left(u, \tilde{\sim}_{0}\right)=\int_{0}^{\infty}\left\{|\mathcal{C} \sim(t)|_{Y}^{2}+\left|\mathcal{R}^{1 / 2} u(t)\right|_{U^{\prime}}^{2}\right\} d t
$$

subject to

$$
\begin{aligned}
& \dot{z}(t)=\mathcal{A} \approx(t)+\mathcal{B} u(t) \\
& z(0)=z_{0} .
\end{aligned}
$$

The positive, self-adjoint operator $\mathcal{R}$ is used to wright various components of the control. 
As detailed in $[2,5]$, if $(\mathcal{A}, \mathcal{B})$ is stabilizable and $(\mathcal{A}, \mathcal{C})$ is detectable, then the optimal control minimizing (3.2) is given by

$$
\bar{u}(t)=-\mathcal{R}^{-1} \mathcal{B}^{*} \Pi z(t)
$$

where $\Pi$ solves the algebraic Riccati equation

$$
\left(\mathcal{A}^{*} \mathrm{II}+\mathrm{II} \mathcal{A}-\Pi \mathcal{B R}^{-1} \mathcal{B}^{*} \Pi+\mathcal{C}^{*} \mathcal{C}\right) z=0 \quad \text { for all } z \in \mathcal{V}
$$

and $\bar{z}(t)=\mathcal{S}(t) \approx 0$. Here $\mathcal{S}(t)$ is the closed loop semigroup generated by $\mathcal{A}-\mathcal{B R}^{-1} \mathcal{B}^{*} I I$.

For implementation purposes, it is necessary to define an approximate system and controls, and determine convergence criteria for these approximate controls when fed back into the infinite dimensional system. The approximations are consiclered in a Galerkin framework with trajectories evolving in the finite dimensional subspaces $\mathcal{V}^{N} \subset \mathcal{V} \subset \mathcal{H}$. It is assumed that the approxination method satisfies the standard convergence conditions

$\left(\right.$ HIN) For any $z \in \mathcal{V}$, there exists a sequence $\tilde{z}^{N} \in \mathcal{V}^{N}$ such that $\left|z-z^{N}\right|_{\mathcal{V}} \rightarrow 0$ as $N \rightarrow \infty$.

The finite dimensional operators and approximating system are then determined as follows. The operator $\mathcal{A}^{N}: \mathcal{V}^{N} \rightarrow \mathcal{V}^{N}$ which approxinates $\mathcal{A}$ is defined by restricting $\sigma$ to $\mathcal{V}^{N} \times \mathcal{V}^{N}$; this yields

$$
\left\langle-\mathcal{A}^{N} \Upsilon, \Lambda\right\rangle_{\mathcal{H}}=\sigma(\Upsilon, \Lambda) \quad \text { for all } \Upsilon . \Lambda \in \mathcal{V}^{N}
$$

For each $N$, the (Co semigronp) on $\mathcal{V}^{N}$ which is generated by $\mathcal{A}^{N}$ is denoted by $\mathcal{T}^{N}(t)$. The control operator is approximated by $\mathcal{B}^{N} \in \mathcal{L}\left(U, \mathcal{V}^{N}\right)$ given by

$$
\left\langle\mathcal{B}^{N} u, \Lambda\right\rangle_{\mathcal{H}}=\left\langle u, \mathcal{B}^{*} \Lambda\right\rangle_{\mathcal{H}} \quad \text { for all } u \in l, \Lambda \in \mathcal{V}^{N}
$$

while $\mathcal{C}^{N}$ denotes the restriction of the observation operator $\mathcal{C}$ to $\mathcal{V}^{N}$. Finally, we let $\rho^{N}$ denote the usual orthogonal projection of $\mathcal{H}$ onto $\mathcal{V}^{N}$ which by clefinition satisfies

$$
\begin{aligned}
& \text { (i) } P^{N} \Upsilon \in \mathcal{V}^{N} \quad \text { for } \Upsilon \in \mathcal{H} \\
& \text { (ii) }\left\langle P^{N} \Upsilon-\Upsilon, \Lambda\right\rangle_{\mathcal{H}}=0 \text { for all } \Lambda \in \mathcal{V}^{N}
\end{aligned}
$$

This projection can be extended to $P^{N} \in \mathcal{L}\left(\mathcal{V}^{*}, \mathcal{V}^{N}\right)$ by replacing the $\mathcal{H}$-inner product $\langle\Upsilon . \Lambda\rangle_{\mathcal{H}}$ by the duality product $\langle\Upsilon . \Lambda\rangle_{\mathcal{V} * \mathcal{V}}$ and considering $\Upsilon \in \mathcal{V}^{*}$.

The approximate problem corresponding to $(2.10)$ with $g \equiv 0$ can then be formulated as

$$
\begin{aligned}
& \frac{d}{d t}\left\langle z^{N}(t), \Lambda\right\rangle_{\mathcal{H}}+\sigma\left(z^{N}(t), \Lambda\right)=\left\langle\mathcal{B}^{N} u(t), \Lambda\right\rangle_{\mathcal{H}} \quad \text { for all } \Lambda \in \mathcal{V}^{N} \\
& z^{N}(0)=P^{N} \approx 0 .
\end{aligned}
$$

This has the solution

$$
z^{N}(t)=\mathcal{T}^{N}(1) P^{N} z_{0}+\int_{0}^{1} \mathcal{T}^{N}(t-s) P^{N} \mathcal{B}^{N} u(s) d s
$$


The following theorems taken from $[2,5]$ can be used to establish the convergence of the approximate gains to their infinite dimensional counterparts for certain classes of shell models (see specifically Theorem 7.10 and Lemma 7.13 of [5]).

Theorem 3. Assume that the injection $i: V \hookrightarrow H$ is compact. Moreover, suppose that the damping sesquilinear form can be decomposed as $\sigma_{2}=\delta \sigma_{1}+\hat{\sigma}_{2}$, for some $\delta>0$, where the continuous sesquilinear form $\hat{\sigma}_{2}$ satisfies for some $\lambda \in \mathbb{R}$

$$
\operatorname{Re} \hat{\sigma}_{2}(\phi, \phi) \geq-\frac{\delta}{2}|\phi|_{r^{r}}^{2}-\lambda|\phi|_{H}^{2} \quad \text { for all } \phi \in V
$$

Finally, suppose that the operator $A_{1}^{-1} \hat{A}_{2}$, where $\hat{A}_{2} \in \mathcal{L}\left(V, V^{*}\right)$ is defined by $\left\langle\hat{A}_{2} \phi, \eta\right\rangle_{V * V}=$ $\hat{\sigma}_{2}(\phi, \eta)$, is compact on $\boldsymbol{l}$.

If for some $\omega \in \mathbb{R}$ and $M \geq 1, \mathcal{T}(t)$ satisfies

$$
|\mathcal{T}(t)|_{\mathcal{L}(\mathcal{H})} \leq M_{c^{\omega t}} \quad, \quad t \geq 0
$$

then for any $\varepsilon>0$ there exists an integer $N_{s}$ such that for $N \geq N_{s}$,

$$
\left|\mathcal{T}^{N}(t) P^{N}\right|_{\mathcal{L}(\mathcal{H})} \leq \widehat{M}_{\epsilon}^{(\omega+\varepsilon) t}, \quad t \geq 0
$$

for some constant $\widetilde{M}>0$ independent of $N$.

Theorem 4. Assume that the injection $i: V \hookrightarrow H$ is compact. Let the sesquilinear form $\sigma$ associated with the first-order system $(2.10)$ be continuous and $\mathcal{V}$-elliptic. Assume that the operators $\mathcal{A}, \mathcal{B}, \mathcal{C}$ of $(2.11),(2.9),(3.1)$, respectively, satisfy: $(\mathcal{A}, \mathcal{B})$ is stabilizable and $(\mathcal{A}, \mathcal{C})$ is detectable where $\mathcal{B} \in \mathcal{L}\left(l, \mathcal{V}^{*}\right)$ is unbounded and $\mathcal{C} \in \mathcal{L}(\mathcal{H}, Y)$ is bounded. Consider an approximation method which satisfies (H1N). Finally, suppose that for fixed $N_{0}$ and $N>N_{0}$. the pair $\left(\mathcal{A}^{N}, \mathcal{B}^{N}\right)$ is uniformly stabilizable and $\left(\mathcal{A}^{N}, \mathcal{C}^{N}\right)$ is uniformly detectable.

Then for $N$ sufficiently large, there exists a unique nonnegative self-adjoint solution $\operatorname{II}^{N} \in$ $\mathcal{L}\left(\mathcal{V}^{*}, \mathcal{V}\right)$ to the $N^{t h}$ approximate algebraic Riccati equation

$$
\mathcal{A}^{N^{*}} \mathrm{II}^{N}+\mathrm{II}^{N} \mathcal{A}^{N}-\mathrm{II}^{N} \mathcal{B}^{N} \mathcal{R}^{-1} \mathcal{B}^{N^{*}} \mathrm{II}^{N}+\mathcal{C}^{N^{*}} \mathcal{C}^{N}=0
$$

in $\mathcal{V}^{N}$. There also exist constants $M_{3} \geq 1$ and $\omega_{3}>0$ independent of $N$ such that $\mathcal{S}^{N}(t)=$ $e^{\left(\mathcal{A}^{N}-\mathcal{B}^{N} \mathcal{R}^{-1} \mathcal{B}^{N^{*}} \Pi^{N}\right) t}$ satisfies

$$
\left|\mathcal{S}^{N}(t)\right|_{\mathcal{V}^{N}} \leq M_{3 \mathrm{r}^{-\omega_{3} t}} \quad, \quad t>0
$$

Moreover, the convergence of the Riccati and control operators

$$
\begin{aligned}
& \Pi^{N} P^{N} \approx \stackrel{s}{\rightarrow} \Pi z \text { in } \mathcal{V} \text { for every } \approx \in \mathcal{V}^{*} \\
& \left|\mathcal{B}^{N^{*}} \Pi^{N} P^{N}-\mathcal{B}^{*} I I\right|_{\mathcal{L}\left(\mathcal{H}, l^{\prime}\right)} \rightarrow 0,
\end{aligned}
$$

as $N \rightarrow \infty$ is obtained. 
Example 1. We consider in this example a shell with constant parameters $\rho, E, \nu, c_{D}$. Such a case would arise if modeling a homogeneous shell or a shell in which the variance of material properties across regions with actuators is negligible. The sesquilinear forms for this mode] are specified in (2.7). Due to the constant coefficients, $\sigma_{2}$ can be written as $\sigma_{2}=\delta \sigma_{1}+\hat{\sigma}_{2}$ where $\delta=\frac{C_{D}}{E}$ and $\hat{\sigma}_{2}(\Phi, \Psi)=\mu \int_{\Gamma_{0}} w \eta_{3} d \gamma$. It follows immediately that

$$
\operatorname{Ri} \hat{\sigma}_{2}(\phi, \phi)=\mu \int_{\Gamma^{\prime}} \phi^{2} d \gamma \geq-\frac{\delta}{2}|\phi|_{V}^{2}
$$

for all $\phi \in V$. The boundedness of the operator $\hat{A}_{2}$ generated by $\hat{\sigma}_{2}$ follows directly from the boundedness of $\hat{\sigma}_{2}$. Furthermore, it is noted that $A_{1}^{-1} \in \mathcal{L}\left(V^{*}, V\right)$ can be written as an operator on $V \rightarrow V$ by $A_{1}^{-1}=1_{1}^{-1} i^{*} i$ where the injections $i: V \hookrightarrow H, i^{*}: H \hookrightarrow I^{*}$ are compact. Thus $A_{1}^{-1}$ is compact on $V$ which implies that $A_{1}^{-1} \hat{A}_{2}$ is compact on $V$ since it is formed from the product of compact and bounded linear operators. Finally, the exponential stability of $\mathcal{T}(t)$, the stabilizability of $(\mathcal{A}, \mathcal{B})$ and the detectability of $(\mathcal{A}, \mathcal{B})$ are guaranteed by Theorem 1. The hypotheses of Theorem 3 are then satisfied for this system and one obtains uniform bounds on the approximating semigroups. The convergence of the Riccati and control operators is then obtained from Theorem 4.

\subsection{Periodic Exogenous Input}

A reasonable assumption in many mechanical systems is that $g$ is periodic in time with period $\tau$. The system to be controlled in this case is

$$
\begin{aligned}
& \dot{z}(t)=\mathcal{A} z(t)+\mathcal{B} u(t)+g(t) \\
& z(0)=z(\tau)
\end{aligned}
$$

and an appropriate quadratic functional to be minimized is

$$
J_{\tau}(u)=\frac{1}{2} \int_{0}^{\tau}\left\{|\mathcal{C} \approx(t)|_{Y}^{2}+\left|\mathcal{R}^{1 / 2} u(t)\right|_{\ell}^{2}\right\} d t
$$

Note that the periodic exogenous term $g$ cau be used to model inputs such as noise generated by rotating engine components (e.g., propellers or turbines) or periodic electromagnetic disturbances.

To guarantee the existence of a unique Riccati solution and control for the system (3.5), it is assumed that $(\mathcal{A}, \mathcal{B})$ is stabilizable and $(\mathcal{A}, \mathcal{C})$ is detectable. Furthermore, it is assumed that $g \in L^{2}(0, \tau ; \mathcal{H})$ and that $\mathcal{B}$ is bounded. Tnder these conditions, it is verified in [6] that the Riccati equation

$$
\mathcal{A}^{*} \mathrm{II}+11 \mathcal{A}+\| \mathcal{B} \mathcal{R}^{-1} \mathcal{B}^{*} I I+\mathcal{C}^{*} \mathcal{C}=0
$$

has a unique solution. Furthermore, if $r$ denotes the $T$-periodic solution of the adjoint or tracking equation

$$
\begin{aligned}
& \dot{r}(1)=-\left[\mathcal{A}-\mathcal{B} \mathcal{R}^{-1} \mathcal{B}^{*} \mid 1\right]^{*} r(1)+\| g(t) \\
& r(0)=r(\tau)
\end{aligned}
$$


and $\bar{z}$ is the closed loop solution of

$$
\begin{aligned}
& \dot{\sim}(t)=\left[\mathcal{A}-\mathcal{B} \mathcal{R}^{-1} \mathcal{B}^{*} \Pi\right] \bar{z}(t)-\mathcal{B} \mathcal{R}^{-1} \mathcal{B}^{*} r(t)+g(t) \\
& \bar{z}(0)=\bar{z}(\tau),
\end{aligned}
$$

then the optimal control is given by

$$
u(t)=-\mathcal{R}^{-1} \mathcal{B}^{*}[\Pi \approx(t)-r(t)] .
$$

The LQR theory for this case is less complete than that for systems with no exogenous input and is currently limited to bounded control inputs $\mathcal{B}$. The synthesis of the theory for unbounded input operators and periodic exogenous forces is currently under investigation. The effectiveness of the method is illustrated in the final example of this work.

\section{Approximation Method}

A Galerkin method was used to approximate the solutions $u, v, u$ to the system (2.6), or equivalently. (2.10). The approximating subspaces were taken of the form $\mathcal{V}^{N}=\operatorname{span}\left\{\mathcal{B}_{u_{k}}\right\} \times$ span $\left\{\mathcal{B}_{v_{k}}\right\} \times$ span $\left\{\mathcal{B}_{w_{k}}\right\}$ where $\mathcal{B}_{u_{k}}, \mathcal{B}_{v_{k}}, \mathcal{B}_{w_{k}}$ denote bases for the $u, v$ and $w^{\prime}$ displacements, respectively. To exploit the tensor nature of the shell domain $\Gamma_{0}$ and periodicity in $\theta$, the bases were constructed with Fourier components in $\theta$ and cubic splines in $x$ (see [7] for details). The approximate displacements were then given by the expansions

$$
\begin{aligned}
u^{N}(t, \theta, x) & =\sum_{k=1}^{\mathcal{N}_{u}} u_{k}(t) \mathcal{B}_{u_{k}}(\theta, x) \\
v^{N}(t, \theta, x) & =\sum_{k=1}^{\mathcal{N}_{n}} v_{k}(t) \mathcal{B}_{v_{k}}(\theta, x) \\
w^{N}(t, \theta, x) & =\sum_{k=1}^{\mathcal{N}_{u}} w_{k}(t) \mathcal{B}_{u_{k}}(\theta, x) .
\end{aligned}
$$

To obtain a finite dimensional system with matrices corresponding to the finite dimensional operators in (3.3) and (3.4), the sesquilinear forms $\sigma_{1}$ and $\sigma_{2}$ were restricted to $\mathcal{V}^{N}$. This yields the matrix system

$$
\begin{aligned}
& {\left[\begin{array}{cc}
K_{E}^{\mathcal{N}} & 0 \\
0 & M^{\mathcal{N}}
\end{array}\right]\left[\begin{array}{l}
\dot{\vartheta}^{\mathcal{N}}(t) \\
\ddot{\vartheta}^{\mathcal{N}}(t)
\end{array}\right]=\left[\begin{array}{cc}
0 & K_{E}^{\mathcal{N}} \\
-K_{E}^{\mathcal{N}} & -K_{r_{D}}^{\mathcal{N}}
\end{array}\right]\left[\begin{array}{c}
\hat{v}^{\mathcal{N}}(t) \\
\dot{\vartheta}^{\mathcal{N}}(t)
\end{array}\right]+\left[\begin{array}{c}
0 \\
\tilde{B}^{\mathcal{N}}
\end{array}\right][u(t)]+\left[\begin{array}{c}
0 \\
\tilde{g}^{\mathcal{N}}(t)
\end{array}\right]} \\
& {\left[\begin{array}{cc}
K_{E}^{\mathcal{N}} & 0 \\
0 & M^{\mathcal{N}}
\end{array}\right]\left[\begin{array}{l}
\vartheta^{\mathcal{N}}(0) \\
\hat{\vartheta}^{\mathcal{N}}(0)
\end{array}\right]=\left[\begin{array}{l}
y_{1}^{\mathcal{N}} \\
y_{2}^{\mathcal{N}}
\end{array}\right]}
\end{aligned}
$$

where $\boldsymbol{y}^{\prime}(t)=\left[u_{1}(t), \cdots, u_{\mathcal{N}_{u}}, v_{1}(t), \cdots, v_{\mathcal{N}_{1}}, w_{1}(t), \cdots, w_{\mathcal{N}_{u}}\right]^{T}$ contains the $\mathcal{N}=\mathcal{N}_{u}+\mathcal{N}_{u}+\mathcal{N}_{n}$ generalized Fourier corfficients. The s patch inputs are contained in $u(t)=\left[u_{1}(t), \cdots, u_{s}(t)\right]^{T}$. The reader is referred to [7] for details concerning the construction of the mass, stiffness and damping matrices $M^{\mathcal{N}} \cdot K_{E}^{\mathcal{N}}, K_{r_{D}}^{\mathcal{N}}$, the inputs $\dot{B}^{\mathcal{N}}, \tilde{g}^{\mathcal{N}}(t)$ and the initial conditions $y_{1}^{\mathcal{N}}, y_{2}^{\mathcal{N}}$. 
Multiplication by the inverted mass matrix yields the ('auchy equation

$$
\begin{aligned}
& \dot{z}^{N}(t)=A^{N} z^{N}(t)+B^{N} u(t)+g^{N}(t) \\
& z^{N}(0)=z_{0}^{N},
\end{aligned}
$$

where $z^{N} \in R^{2 \mathcal{N}}=\left[\vartheta^{\mathcal{N}}(t), i \mathcal{N}^{\mathcal{N}}(t)\right]^{T}$. This system forms the constraint equations used in the finite dimensional LQR thoory discussed in Section :?.

\section{$5 \quad$ Numerical Example}

We consider here an exogenous force $g$ which is periodic in time with period $\tau=1000 \pi$ $(500 \mathrm{~Hz})$. The distribution of the force was taken to be binormal in the transverse and longitudinal directions and centered at $(x, \theta)=(\ell / 2, \theta)$ and $(x, \theta)=(f / 2, \pi)$ as depicted in Figure 2. The magnitude of the 1ransverse component $\hat{q}_{n}$ was one lundred times that of the longitudinal component $\hat{q}_{r}$ so as to model an input consisting primarily of acoustic sources located adjacent to $(f / 2,0)$ and $(/ / 2, \pi)$.

Six pairs of piezoceramic patches of length $1 \mathrm{~cm}$ and radial measure $\pi / 3$ were employed as actuators. The locations and material properties of the patches along with the dimensions and physical parameters for the shell are summarized in Table 1.

To accommodate the periodic exogenous force $g$, control inputs to the twelve patches were computed using the feedback law (3.6). Note that in this formulation, independent voltages are determined for the individual patches. This provides the capability of generating both inplane forces and bending moments in the regions covered by the patches so that longitudinal. circumferential and transverse vibrations can be controlled.

Time histories of the uncontrolled and controlled shell displacements at the point $p_{1}=$ $(3 / / 4, \pi / 32)$, depicted in Figure 2, are plotted in Figure 3. The open loop trajectories exhibit both a transient response settling into steady state and a beat phenomenon due to the close proximity of the driving frequency and natural frequencies for the shell. At this observation

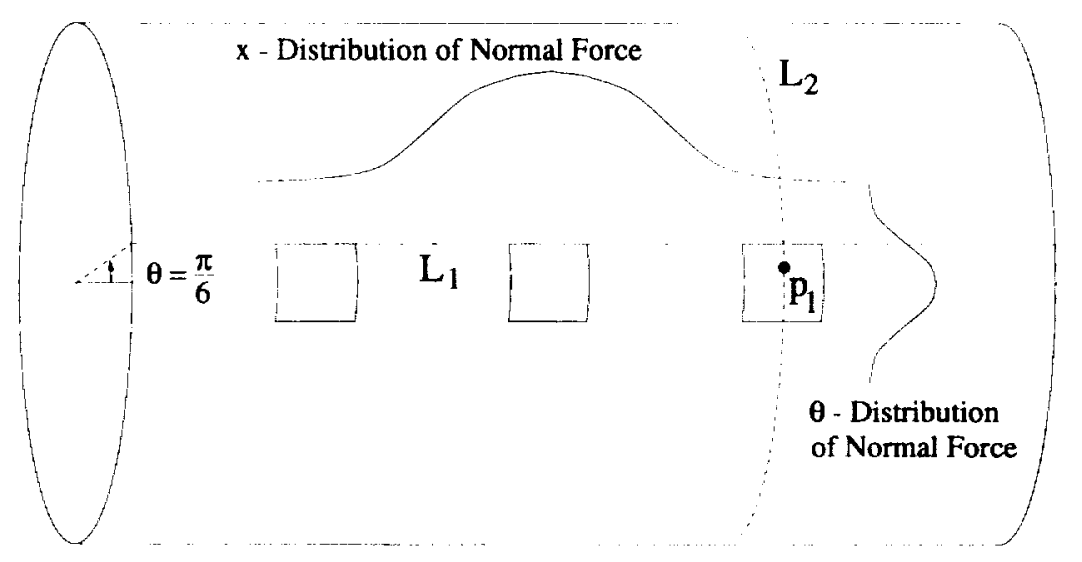

Figure 2. Distribution of nomal forcing function at $\theta=0$ and $\theta=\pi$. Observation liness $L_{1}=\{(x, \theta) \mid 0 \leq x \leq \ell, \theta=\pi / 6\}, L_{2}=\{(x, \theta) \mid r=3 f / 4.0 \leq \theta \leq 2 \pi\}$ and olservation point $p_{1}=(3(/ 4, \pi / 32)$. 
point, all three displacement components are reduced by more than $90 \%$ when controlling voltages are fed back to the patches.

To illustrate the spatial attenuation due to the feedback of voltages to the patches, root mean square (rms) plots of the uncontrolled and controlled trajectories along the axial line $L_{1}$ and circumferential line $L_{2}$ (see Figure 2) are plotted in Figure 4 and 5, respectively. For the open loop case, these plots illustrate a standing wave in all three components of the displacement (the slight asymmetry in the axial plots is due to the longitudinal input $\hat{q}_{x}$ ). The figures also demonstrate significant reductions in all three displacement levels, even in regions not covered by patches. This further illustrates the effectiveness through which the model-based control law can be used to attenuate shell vibrations.

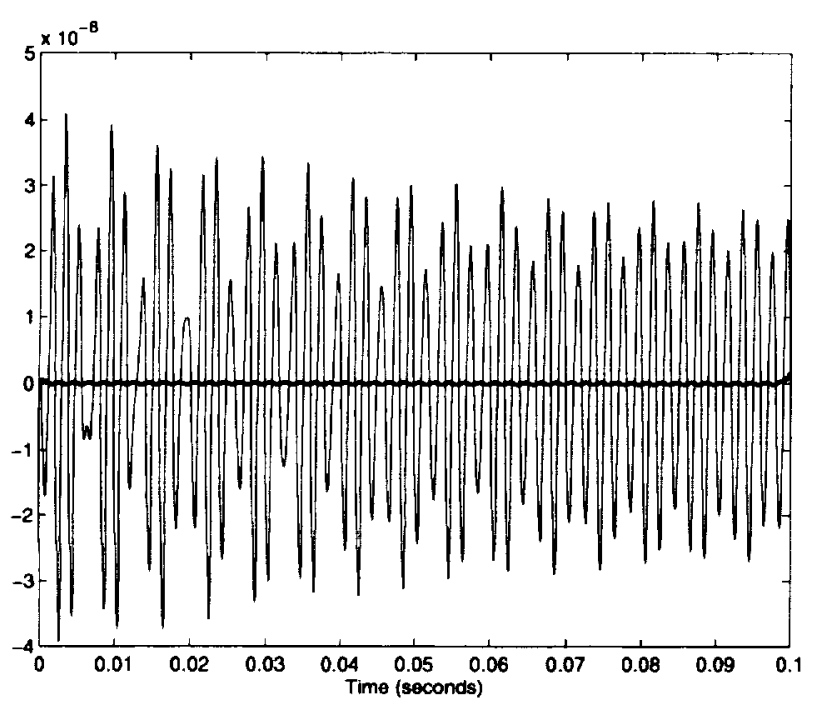

(a)

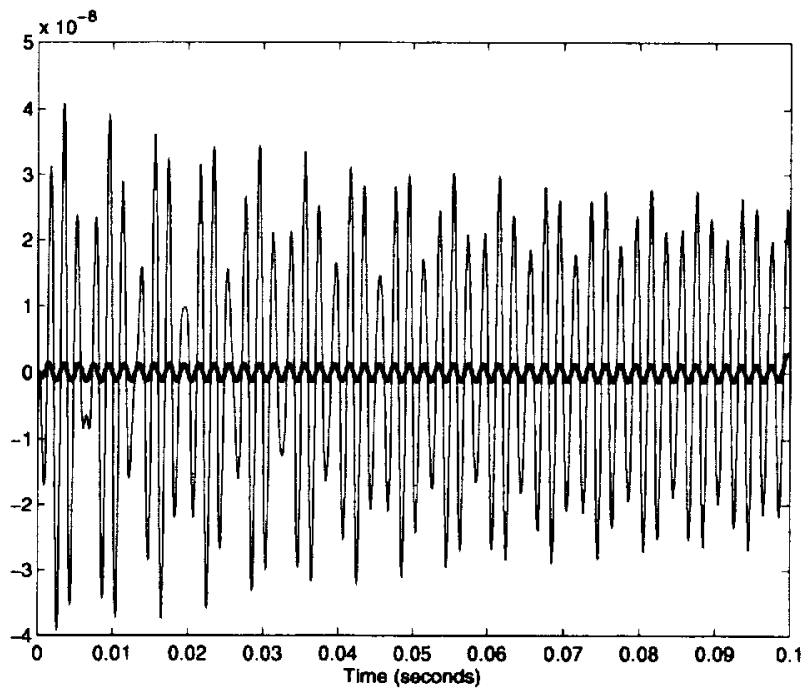

(b)

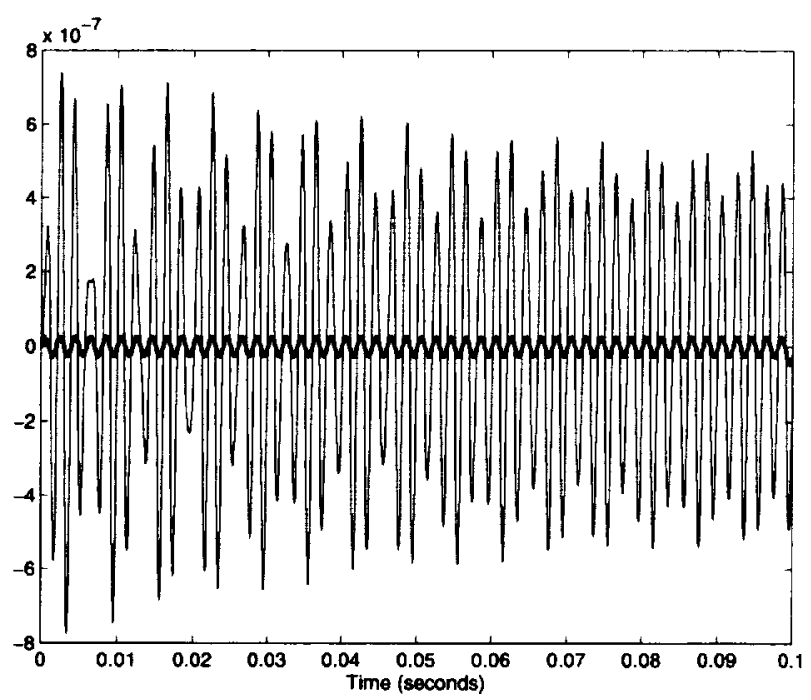

(c)

Figure 3. Incontrolled and controlled shell displacements at the point $p_{1}=(3 / / 4, \pi / 32)$; (a) longitudinal $u$, (b) circumferential $v$. (c) transverse $w$ displacenents; _ (uncontrolled), - (controlled). 

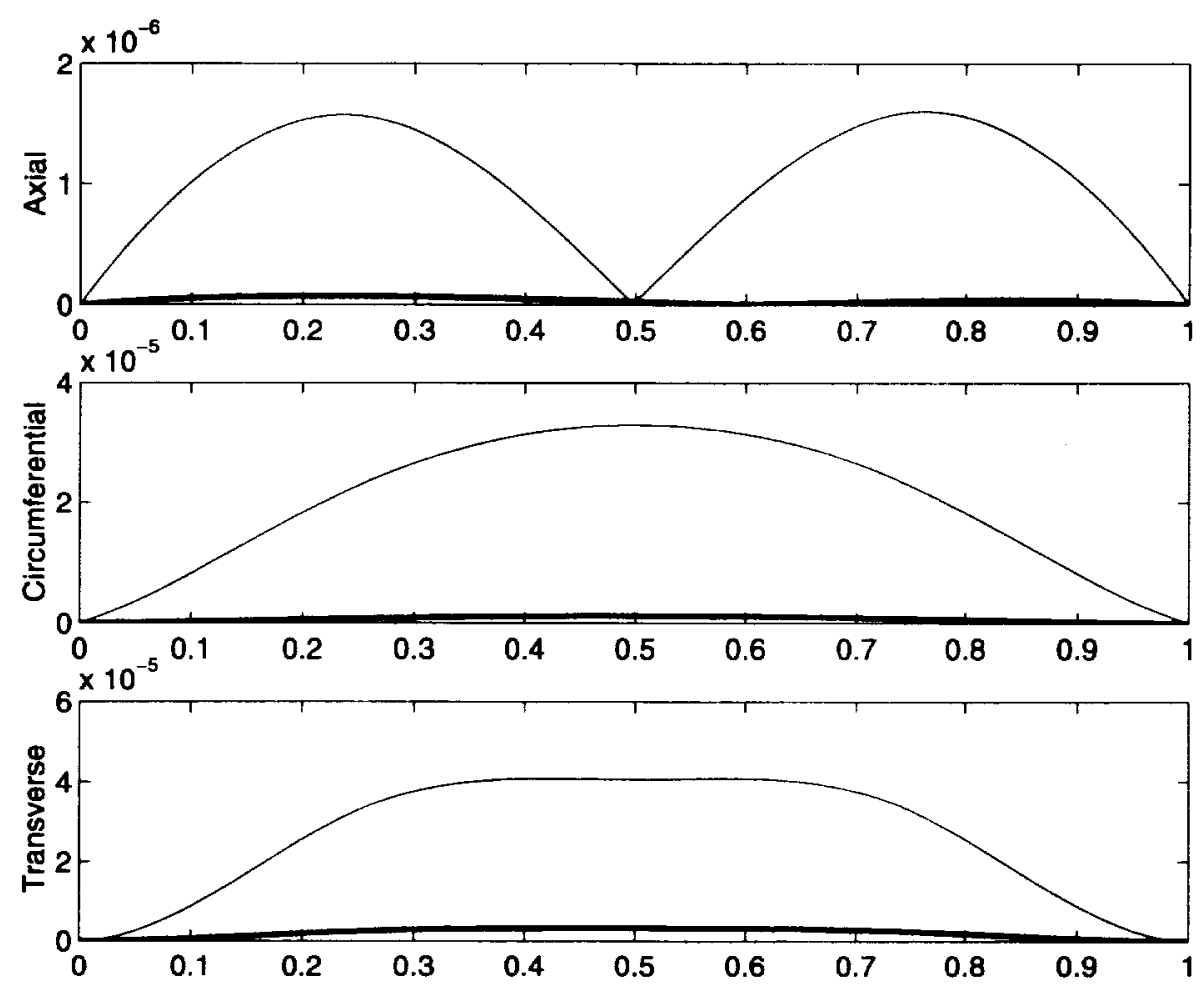

Figure 4. Root mean square (rms) displacements along the axial line $L_{1}$; — (uncontrolled), (controlled).
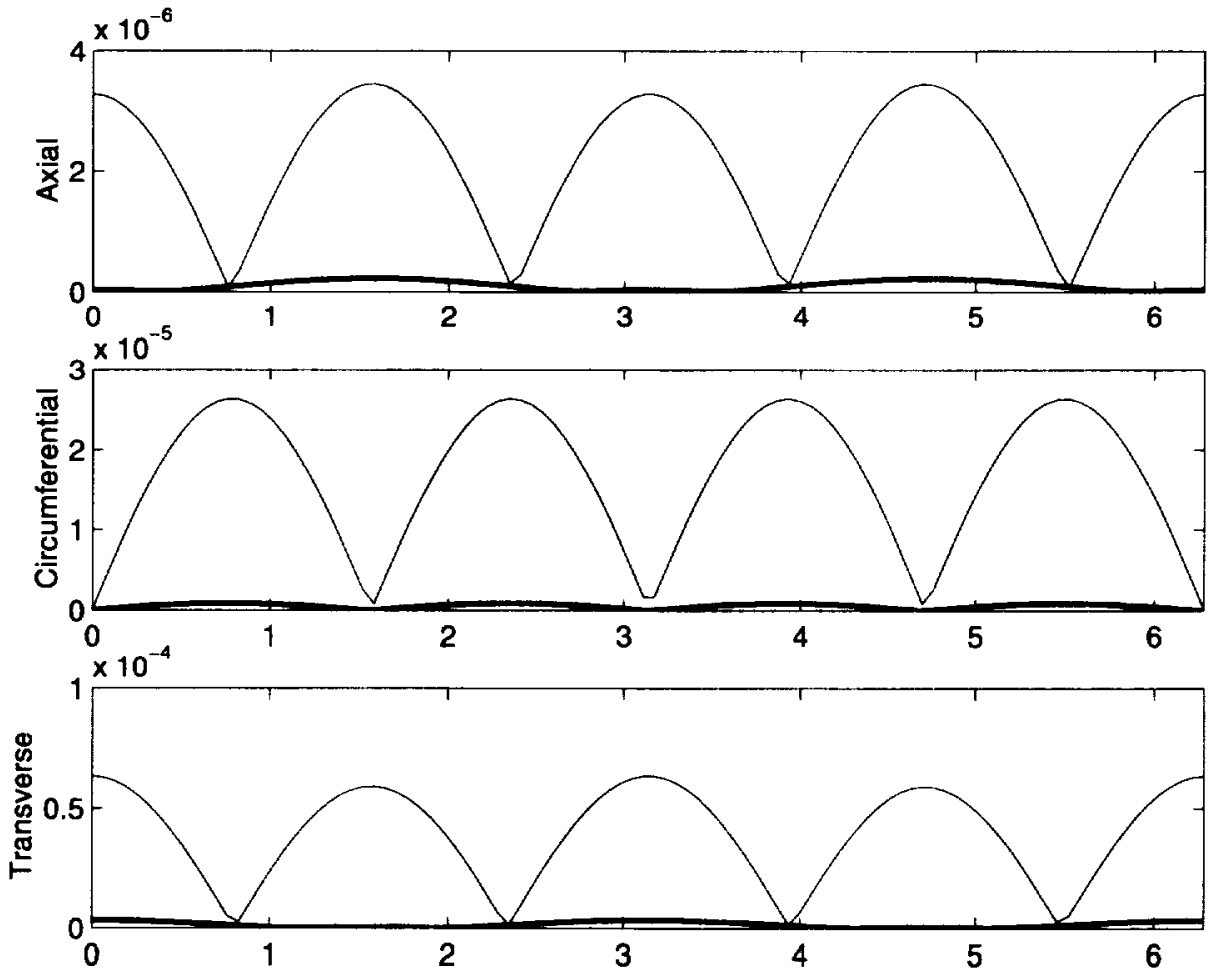

Figure 5. Root mean square (rms) displacements along the circumferential line $L_{2}$ : - (uncontrolled), (controlled). 


\begin{tabular}{|c|c|c|}
\hline & Dimensions & Parameters \\
\hline Shell & $\begin{array}{l}h=.00127 \mathrm{~m} \\
R=.4 \mathrm{~m} \\
r=1 \mathrm{~m}\end{array}$ & $\begin{array}{l}\rho=2700 \mathrm{~kg} / \mathrm{m}^{3} \\
E=7.1 \times 10^{10} \mathrm{~N} / \mathrm{m}^{2} \\
c_{D}=1.47 \times 10^{5} \mathrm{Nms} \\
\nu=.33 \\
\mu=58.97 \mathrm{Ns} / \mathrm{m}^{2}\end{array}$ \\
\hline Patches & $\begin{aligned} & h_{p e}=.0001778 \mathrm{~m} \\
& \text { Centers }(x, 0):(.25,0),(.5,0),(.75,0) \\
&(.25, \pi),(.5, \pi),(.75, \pi) \\
& \text { Dimensions: } x: 0.1 \mathrm{~cm}, \theta: \pi / 3\end{aligned}$ & $\begin{array}{l}\rho_{p \epsilon}=7600 \mathrm{~kg} / \mathrm{m}^{3} \\
E_{p \epsilon}=6.3 \times 10^{10} \mathrm{~N} / \mathrm{m}^{2} \\
c_{D_{p e}}=1.7 \times 10^{5} \mathrm{Nm} \\
\nu_{p \epsilon}=.31 \\
d_{31}=190 \times 10^{-12} \mathrm{~m} / \mathrm{V}\end{array}$ \\
\hline
\end{tabular}

Table 1. Dimensions and physical parameters for the shell and patches.

\section{Conclusions}

A model-based LQR method for controlling shell vibrations has been presented here. While developed in the context of a modified Donnell-Mushtari cylindrical shell model. the method is quite general and can be directly extended to other models and geometries. Inder the assumption of strong or lielvin-Voigt damping (a reasonable and typical assumption for many shell materials), model well-posedness and convergence of control gains is obtained using analytic semigroup theory combined with LQR results for unbounded input operators.

The Galerkin method used to approximate the system dynamics utilizes bases constructed from tensored Fourier polynomials and modified cubic splines. As discussed in [7], care must be taken when developing methods for approximating shell dynamics so as to avoid shear or membrane locking. One manifestation of locking is the existence of model dynamics which are incorrectly approximated by the numerical method. The use of a numerical method which exhibits locking can lead to a loss of control authority and potential controller destabilization if the approximations are sufficiently inaccurate. Further details regarding issues concerning the approximation of shell dynamics and convergence properties of the numerical method can be found in $[T]$.

The numerical example demonstrates the effectiveness of the model-based control method for attenuating all three components of the shell displacement in the presence of both transient and steady state dynamics. Furthermore, by modeling the global shell dynamics and patch interactions through coupled PDE and constructing the control law in terms of these PDE, significant reductions in displacement levels throughout the shell are obtained, even in regions devoid of patches. Numerical implementation of the LQR method in this manner provides a first step toward the development of model-based state estimators and compensators which can be experimentally implemented in shell applications.

\section{Acknowledgements}

The authors thank H.T. Banks, CRSC: North (arolina State University for input regarding various aspects of this investigation. 


\section{References}

[1] H.T. Banks and K. Ito, "A Unified Framework for Approximation in Inverse Problems for Distributed Parameter Systems," Control-Theory and Advanced Technology, 4, pp. 73-90, 1988 .

[2] H.T. Banks and K. Ito, "Approximation in LQR Problems for Infinite Dimensiona] Systems with Unbounded Input Operators," ('RSC: Technical Report CRSC-TR94-22, November 1994; Journal of Mathcmatical Systems, Estimation and Control, to appear.

[3] H.T. Banks, R.C. Smith and Y. Wang, "The Modeling of Piezoceramic Patch Interactions with Shells, Plates. and Beams," Quarterly of Applied Mathematics, 53(2), pp. 35.3-381, 1995.

[4] H.T. Banks, R.C. Smith and Y. Wang, "Modeling and Parameter Estimation for an Imperfectly Clanped Plate," CRSC: Technical Report ('RS('-TR95-2, 1995; Computution and Control IT, (K.L. Bowers and J. Lund, eds.), Birkläuser, Boston, 1995. pp. 23-42.

[5] H.T. Banks, R.('. Smith and Y. Wang, Smart Material Structures: Modeling. Estimation and Control, Masson/John Wiley, Paris/C'hichester, 1996.

[6] G. Da Prato, "Synthesis of Optimal (ontrol for an Infinite Dinensional Periodic Problem," SLAM .J. Control and Optimization, 25(3). 1987, pp. 706-714.

[7] R. del Rosario and R.('. Smith, "Spline Approximation of Thin Shell Dynamics," ICASE Report 96-26, March 1996: International Journal for Numerical Methods in Enginefring, to appear.

[8] ('.R. Fuller, S.D). Snyder, ('.H. Hansen and R.J. Silcox. "Active ('ontrol of Interior Noise in Model Aircraft Fuselages Using Piezoceramic Actuators." Paper 90-392.2, AIAA 13th Aeroacoustics ('onference, Tallahassee, FL, October 1990.

[9] A.W. Leissa, Vibration of Shells, NASA SP-288, 1973; Reprinted by the Acoustical Society of America through the American Institute of Physics. 199:3.

[10] Lester and Lefebvre, "Piezoelectric Actuator Models for Active Sound and Vibration Control of (ylinders," Proceedings of the Conference on Recent Advance in Active Control of Sound and Vibration. Blacksburg, VA, 1991. pp. 3-26.

[11] A. Pazy, Semigroups of Linear Operators and Applications to Partial Differential Equations, Springer-Verlag, New York, 198:3.

[12] R.F. Showalter, Hilbert Space Methods for Partial Diffremtial Equations. Pitman Publishing Ltd., Iondon. 1977.

[1:3] R..J. Silcox, S. Iefebvre. V.L. Metcalf, T.B. Beyer and (.R. Fuller, "Evaluation of Piezoceramic Actuators for control of Aircraft Interior Noise," Proceedings of the DOGLR/AIAA 14th Acroacoustics (onference. Aachen. Germany, May 11-14, 1992.

[14] H. Tanabe, Equations of Evolution. Pitman Publishing Lul.. London, 1979. 



\begin{tabular}{|c|c|c|c|}
\hline \multicolumn{3}{|c|}{ REPORT DOCUMENTATION PAGE } & $\begin{array}{l}\text { Form Approved } \\
\text { OMB No 0704-0188 }\end{array}$ \\
\hline \multicolumn{4}{|c|}{ 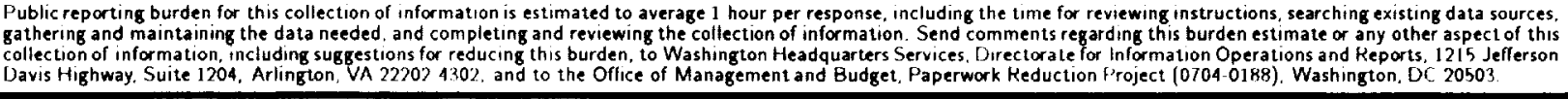 } \\
\hline 1. AGENCY USE ONLY(Leave blank) & $\begin{array}{l}\text { 2. REPORT DATE } \\
\text { March } 1997\end{array}$ & \multicolumn{2}{|c|}{$\begin{array}{l}\text { 3. REPORT TYPE AND DATES COVERED } \\
\text { ('ontractor Report }\end{array}$} \\
\hline \multicolumn{3}{|c|}{$\begin{array}{l}\text { 4. TITLE AND SUBTITLE } \\
\text { LQR control of shell vibrations via piczoceramic actuators }\end{array}$} & \multirow[t]{2}{*}{$\begin{array}{l}\text { 5. FUNDING NUMBERS } \\
\text { (: NAS1-19480 } \\
\text { WU } 505-90-52-01\end{array}$} \\
\hline \multicolumn{3}{|l|}{$\begin{array}{l}\text { 6. AUTHOR(S) } \\
\text { R.C.H. del Rosario } \\
\text { R.C.Smith }\end{array}$} & \\
\hline \multicolumn{3}{|c|}{$\begin{array}{l}\text { 7. PERFORMING ORGANIZATION NAME(S) AND ADDRESS(ES) } \\
\text { Institute for Computer Applications in Sicience and Engineering } \\
\text { Mail Stop 403, NASA Langley Research Center } \\
\text { Hampton, VA } 23681-0001\end{array}$} & $\begin{array}{l}\text { 8. PERFORMING ORGANIZATION } \\
\text { REPORT NUMBER } \\
\text { IC.ASE: REPOIT No. } 97-19\end{array}$ \\
\hline \multicolumn{3}{|c|}{$\begin{array}{l}\text { 9. SPONSORING/MONITORING AGENCY NAME(S) AND ADDRESS(ES) } \\
\text { National Aeronautics and Space Administration } \\
\text { Langley Research Center } \\
\text { Hampton, VA 23681-0001 }\end{array}$} & $\begin{array}{l}\text { 10. SPONSORING/MONITORING } \\
\text { AGENCY REPORT NUMBER } \\
\text { NASA CR-201673 } \\
\text { ICASE Report No. } 97-19\end{array}$ \\
\hline \multicolumn{4}{|c|}{$\begin{array}{l}\text { 11. SUPPLEMENTARY NOTES } \\
\text { Langley Technical Monitor: Dennis M. Bushnell } \\
\text { Final Report } \\
\text { Submitted to Journal of Fluid Mechanics }\end{array}$} \\
\hline \multicolumn{2}{|c|}{$\begin{array}{l}\text { 12a. DISTRIBUTION/AVALABILITY STATEMENT } \\
\text { Unclassified Inlimited } \\
\text { Subject Category } 64\end{array}$} & 12b. DISTf & 12b. DISTRIBUTION CODE \\
\hline \multicolumn{4}{|c|}{ 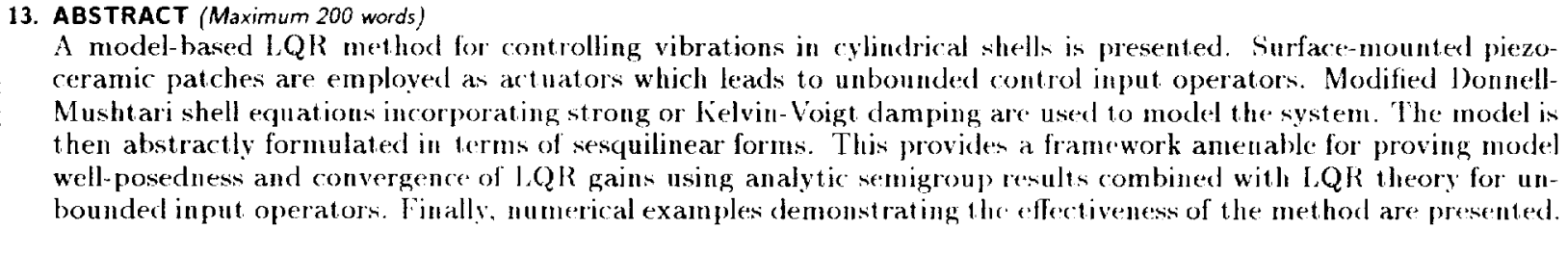 } \\
\hline \multirow{2}{*}{\multicolumn{3}{|c|}{$\begin{array}{l}\text { 14. SUBJECT TERMS } \\
\text { LQR feedback control; thin shell model: (ialerkin approximation }\end{array}$}} & $\begin{array}{l}\text { 15. NUMBER OF PAGES } \\
\qquad 20\end{array}$ \\
\hline & & & $\begin{array}{c}\text { 16. PRICE CODE } \\
\mathrm{A} 0.3 \\
\end{array}$ \\
\hline $\begin{array}{l}\text { 17. SECURITY CLASSIFICATION } \\
\text { OF REPORT } \\
\text { Unclassified }\end{array}$ & $\begin{array}{l}\text { 18. SECURITY CLASSIFICATION } \\
\text { OF THIS PAGE } \\
\text { Indassified }\end{array}$ & $\begin{array}{l}\text { 19. SECURITY CLASSIFICATION } \\
\text { OF ABSTRACT }\end{array}$ & $\begin{array}{l}\text { 20. LIMITATION } \\
\text { OF ABSTRACT }\end{array}$ \\
\hline
\end{tabular}

